\title{
Effect of Fluoride and Chlorhexidine Digluconate Mouthrinses on Plaque Biofilms
}

\author{
Per Rabe $^{1, *}$, Svante Twetman ${ }^{1,2}$, Bertil Kinnby ${ }^{3}$, Gunnel Svensäter ${ }^{3}$ and Julia R. Davies ${ }^{3}$
}

\author{
${ }^{1}$ Maxillofacial Unit, Halland Hospital, SE-301 85, Halmstad, Sweden; ${ }^{2}$ Department of Odontology, Section of Cariology \\ and Endodontics, Faculty of Health and Medical Sciences, University of Copenhagen, Denmark; ${ }^{3}$ Department of Oral \\ Biology, Faculty of Odontology, Malmö University, SE-206 05, Malmö, Sweden
}

\begin{abstract}
Objective: To develop a model in which to investigate the architecture of plaque biofilms formed on enamel surfaces in vivo and to compare the effects of anti-microbial agents of relevance for caries on biofilm vitality. Materials and Methodology: Enamel discs mounted on healing abutments in the pre-molar region were worn by three subjects for 7 days. Control discs were removed before subjects rinsed with $0.1 \%$ chlorhexidine digluconate $(\mathrm{CHX})$ or $0.2 \%$ sodium fluoride $(\mathrm{NaF})$ for 1 minute. Biofilms were stained with Baclight Live/Dead and z-stacks of images created using confocal scanning laser micoscopy. The levels of vital and dead/damaged bacteria in the biofilms, assessed as the propor-

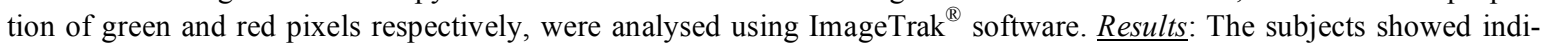
vidual differences in biofilm architecture. The thickness of the biofilms varied from $28-96 \mu \mathrm{m}$ although cell density was always the greatest in the middle layers. In control biofilms, the overall levels of vitality were high (71-98\%) especially in the area closest to the enamel interface. Rinsing with either $\mathrm{CHX}$ or $\mathrm{NaF}$ caused a similar reduction in overall vitality. CHX exerted an effect throughout the biofilm, particularly on the surface of cell clusters whereas NaF caused cell damage/death mainly in the middle to lower biofilm layers. Conclusion: We describe a model that allows the formation of mature, undisturbed oral biofilms on human enamel surfaces in vivo and show that CHX and NaF have a similar effect on overall vitality but differ in their sites of action.
\end{abstract}

Keywords: Anti-microbial agents, confocal microscopy, dental plaque, dental implants, enamel, vitality.

\section{INTRODUCTION}

The resident oral microflora is found in the form of surface-associated communities, or biofilms. Oral biofilm formation is initiated by the adhesion of early colonizers to salivary proteins in the enamel pellicle or to the salivaderived films on mucosal surfaces. Upon binding, these pioneer bacteria themselves provide adherence sites and modify the environment allowing later-colonizing species to become established [1]. On the tooth enamel, stabilization of these heterogeneous micro-colonies through synthesis of polysaccharides and proteins, as well as sequestration of host proteins, gives rise to complex microbial communities known as dental plaque. Identification of the initial colonizing species on enamel in vivo shows that the predominant species are streptococci, including Streptococcus oralis, Streptococcus mitis biovar 2 and Streptococcus sanguinis [2] and actinomycetes, including Actinomyces naeslundii.

Microorganisms growing in biofilms have been shown to be highly resistant to antimicrobial agents and can be up to 1000 times more resistant than their planktonic counterparts [3]. This property has, in part, been attributed to poor penetration of antimicrobial substances into biofilms due to the presence of the polymer matrix. However, biofilm bacteria

*Address correspondence to this author at the Maxillofacial Unit, Halland Hospital, SE-301 85, Halmstad, Sweden; Tel: +46 35134050;

E-mail: Per.Rabe@regionhalland.se are also intrinsically less susceptible to antimicrobial substances due to their slow rates of growth and the adoption of a distinct biofilm phenotype, which differs from that of planktonic bacteria. When penetration is poor, bacteria in biofilms may be exposed to sub-lethal concentrations of biocides, contributing to the development of antimicrobial resistance.

The commensal oral microflora is important for health but oral biofilms are also an essential component in the etiology of dental caries. In caries, metabolism of rapidly fermentable carbohydrates results in the production of organic acids in oral biofilms. The low $\mathrm{pH}$ resulting from this process disrupts the mineral equilibrium between the tooth surface and the surroundings leading to demineralization. Approaches aimed at preventing dental caries include mechanical removal of biofilms, application of fluoride, the use of antimicrobial agents such as chlorhexidine gluconate (CHX) and dietary control. Current evidence suggests that topical application of fluoride in the form of toothpaste, gels, varnishes and mouthrinses can reduce caries development (for a review see [4]). Fluoride has been thought to act primarily through the formation of fluorapatite crystals, which have a greater resistance to organic acids than hydroxyapatite. However more recently, fluoride has also been shown to be capable of reducing organic acid production in cariogenic bacteria such as Streptococcus mutans growing in biofilms [5]. 
The cationic bisguanidine, CHX, which has long been used as an antibacterial agent in the oral cavity, has been shown to significantly reduce amounts of dental plaque compared to placebo [6]. The particular sensitivity of S. mutans to CHX has led to its use in the prevention of caries [7]. Although the mechanism of action is not fully elucidated, CHX has also been shown to inhibit both acid production and sugar transport in oral streptococci [8].

Caries often occurs at inaccessible sites where mature biofilms are present. Such biofilms contain, not only mixed bacterial species, but also high levels of biofilm matrix components. Therefore an ability to penetrate into plaque biofilms and reach concentrations required for a bacteriocidal effect is critical if substances are to be effective in caries prevention and treatment. In this study, we have investigated the thickness and architecture of intact plaque biofilms from different individuals, formed on enamel surfaces over 7 days in vivo. We have then tested the effect of rinsing in vivo with sodium fluoride $(\mathrm{NaF})$ and $\mathrm{CHX}$ on bacteria at different levels within the biofilms. The results of this study will shed light upon the ability of the antimicrobial agents, fluoride and CHX to exert an effect in the clinical setting.

\section{MATERIALS AND METHODOLOGY}

\section{Experimental Conditions}

Three healthy volunteers (two female and one male, aged $68-83$ yrs, median 75 years) with at least two single implants in the same premolar region were enrolled in the study. All subjects gave their informed consent and the study was approved by the Regional Ethical Review Board in Lund, Sweden (Dnr 2010/525). The patients were initially referred to the periodontal section of the Maxillofacial Unit Halland Hospital, Sweden for care and had implants installed when their periodontal disease had been sucessfully treated. They retained their normal dietary and oral hygiene habits and were instructed not to brush the abutments or use any chemical plaque control or fluoride rinses during the study period. None of the subjects used tobacco and all displayed a good level of oral hygiene.

\section{Specimen Preparation}

Two premolar implants located in the same quadrant were selected as study sites. The suprastructure was removed from the implants and replaced with specially prepared healing abutments $(\varnothing 4.8 \mathrm{~mm}$, Straumann AG, Basel, Switzerland). An enamel disc recovered from intact extracted human third molars was mounted on the top of each abutment with aid of an orthodontic resin (Smart Bond, Gestrenco international, Gothenburg, Sweden). The enamel specimen had been prepared with diamond burs and abrasive paper in order to achieve a flat surface at a $90^{\circ}$ angle to the axis of the healing abutment.

\section{Biofilm Formation}

After application of the abutments (Fig. 1), a natural biofilm was allowed to develop for 7 days. After this time one of abutments, selected at random, was removed and the patient was instructed to rinse their mouth with $10 \mathrm{ml}$ of the assigned test solution; either $0.1 \%$ CHX (Hexident ${ }^{\circledR}$, Meda
$\mathrm{AB}$ Solna, Sweden) or $0.2 \% \mathrm{NaF}\left(\right.$ Dentan $^{\circledR}$, Meda $\mathrm{AB}$, Solna, Sweden). The rinsing time was one minute followed by a 10 second tap water rinse. Immediately thereafter, the second abutment was removed. The abutments were kept in a moist chamber and the biofilms analysed within 4 hours after removal.

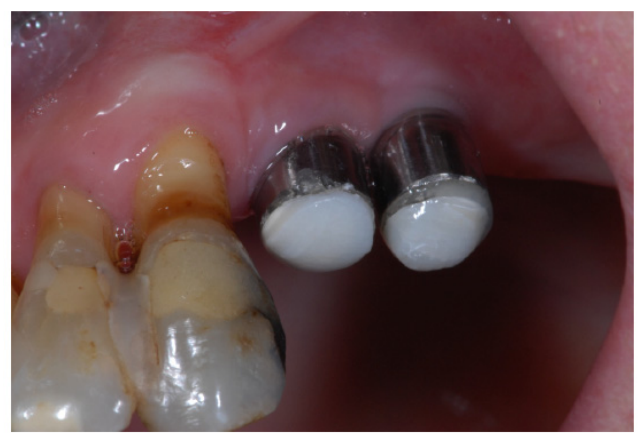

Fig. (1). Photograph showing experimental set-up. Enamel discs were mounted onto healing abutments using orthodontic resin and the abutments placed in the same quadrant for 7 days.

\section{Visualization of Biofilms and Image Analysis}

The samples were investigated using an inverted confocal laser-scanning microscope (CLSM) (Eclipse TE2000, Nikon Corporation, Tokyo, Japan). A custom-built device was developed for the mounting of the healing abutment in the microscope. Green fluorescence was provided by an Ar laser (488 $\mathrm{nm}$ laser excitation) and red fluorescence was given by a G-HeNe laser (543 nm laser excitation). CLSM images were acquired with an oil immersion objective (x60). Ten random image stacks containing optical sections of $2 \mu \mathrm{m}$ (zstep) with a substratum coverage field area of $215 \times 215 \mu \mathrm{m}$ were obtained from each biofilm. Images were recorded with Nikon NIS-Elements software and saved as ics/ids files. The ics/ids files were imported into ImageTrak ${ }^{\circledR}$ software (P. K. Stys; http://www.ucalgary.ca/styslab/ imagetrak) and the number of red and green fluorescent pixels in each optical section analysed to allow calculation of the total biomass as well as the proportion of vital cells. Three-dimensional renditions of representative biofilms were performed using ICY software (Quantitative Image Analysis Unit at the Institut Pasteur; http://www.bioimageanalysis.org/).

\section{RESULTS}

In this study, biofilms were allowed to form on human enamel discs mounted on healing abutments in the oral cavity of three patients. After removal, the biofilms were visualized and image stacks obtained using a CLSM. The total biomass, calculated as the sum of green and red fluorescent pixels, revealed that the three subjects accumulated similar amounts of plaque over the seven-day test period. In subjects 1 and 3, the total biomass on the enamel surface was $4.5 \mathrm{x}$ $10^{6}$ arbitrary units $/ \mathrm{mm}^{2}$ and for subject 2 , the total biomass was $6.5 \times 10^{6}$ arbitrary units $/ \mathrm{mm}^{2}$ enamel (Fig. 2). Representative 3-dimensional images created from z-stacks for each subject are shown in Fig. (2). Comparison of the biofilm height showed that the biofilm from subject 1 was the thinnest ( $28 \mu \mathrm{m}$ thick) while that from subject 3 was more than 3 times as thick $(96 \mu \mathrm{m})$. The fact that these two biofilms 


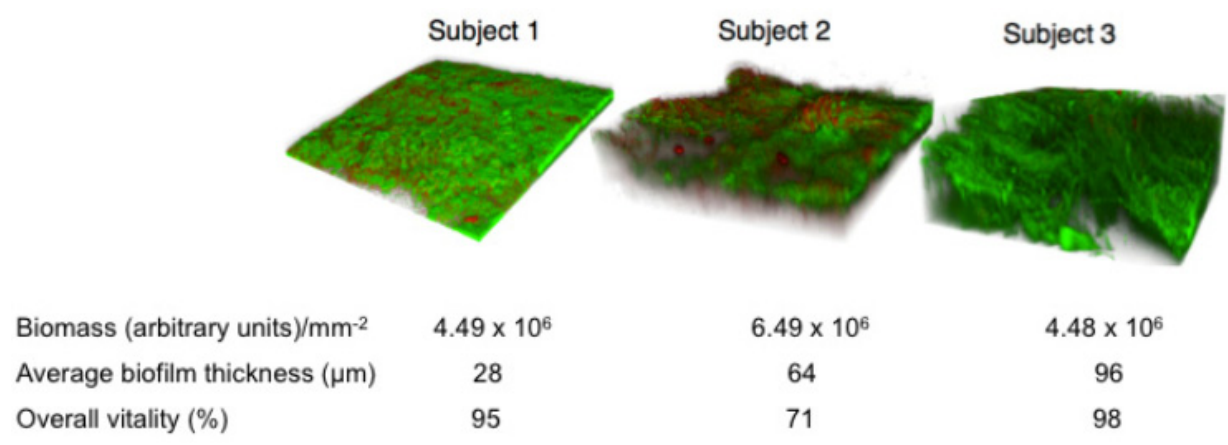

Fig. (2). Architecture of oral biofilms formed on enamel discs in situ in three subjects. Biofilms were allowed to develop on the enamel surfaces mounted on healing abutments in the oral cavity for 7 days. The abutments were then removed, stained with BacLight Live/Dead staining and visualised using CSLM.
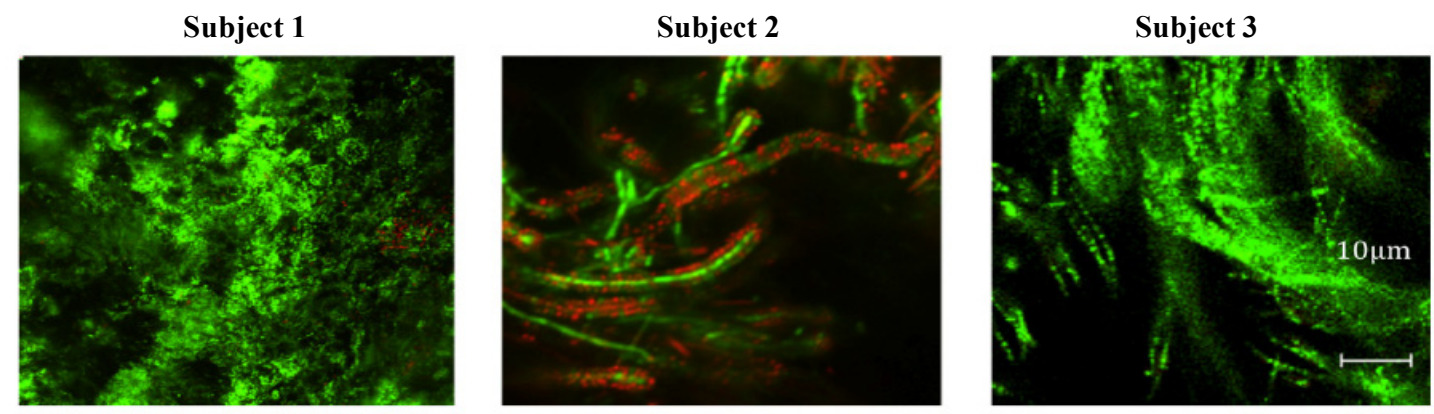

Fig. (3). Structure of the in situ biofilms. Biofilms were stained with BacLight Live/Dead stain and high magnification images taken to allow visualization of the microbial composition. The scale bar represents $10 \mu \mathrm{m}$.

contained the same biomass indicates that the biofilm from subject 3 contained more extracellular space than that from subject 1 . Investigation of the overall vitality within the biofilms (estimated as the percentage of green pixels) showed that subjects 1 and 3 both had high levels of vital biomass (95 and 98\% respectively) whereas this figure was somewhat lower in subject $2(71 \%)$.

Closer investigation of the architecture of the biofilms also revealed differences between the three subjects. In subject 1 the biofilm was dominated by spherical cells, most likely corresponding to streptococci (Fig. 3). In contrast, the biofilm from subject 3 was dominated by long, filamentous bacteria, while that from subject 2 had similarities to both those of subject 1 and subject 3, containing spherical cells that were apparently attached to larger rods. Taken together, the data indicate that the biofilm from subject 1 had a compact structure with mainly spherical cells and low levels of extracellular space. In contrast, biofilms from subjects 2 and 3 were thicker but with the same levels of bacterial biomass as subject 1 , consistent with a more open architecture containing sparsely distributed spherical cells and/or filamentous rods.

To investigate vitality at different levels in the biofilms, the number of green and red pixels in each optical section in the z-stacks was plotted against biofilm level (Fig. 4). For all subjects, the highest biomass was found towards the middle of the biofilm and cell density then decreased towards the external aspects at the enamel and salivary interfaces. The vitality was always high (96-99\%) in the area closest to the enamel interface but the distribution of dead/damaged cells differed between the subjects. Subject 3 had negligible levels of red cells, while in subject 1 the highest proportion of red staining was seen in the middle of the biofilm and in subject 2 this was concentrated towards the middle and upper biofilm levels.

To investigate the effects of antimicrobial agents, biofilms formed over seven days in subject 1 were subjected to rinsing in situ with $0.1 \% \mathrm{CHX}$ or $0.2 \% \mathrm{NaF}$ for 1 minute followed by rinsing with tap water. The number of green and red pixels in each optical section in the z-stacks was then plotted against biofilm level (Fig. 5). After treatment, the biomass of the biofilms was slightly reduced $\left(0.82 \times 10^{6}\right.$ arbitrary units $/ \mathrm{mm}^{2}$ for $\mathrm{CHX}$ and $0.87 \times 10^{6}$ arbitrary units $/ \mathrm{mm}^{2}$ for $\mathrm{NaF}$ ) compared to control. This suggests that the rinsing procedure removed the biofilms from the enamel surfaces to a small extent but that neither of the substances had a major removal effect. $\mathrm{CHX}$ and $\mathrm{NaF}$ both caused an overall reduction in vitality of the total biomass (from $95 \%$ in the control to $63 \%$ ). After the CHX rinse, dead/damaged cells were seen throughout the biofilm and image analysis indicated that antimicrobial effect was the greatest at the cell/liquid interface of the fluid-filled channels running from top to bottom within the structure. Sodium fluoride however, appeared to penetrate and exert an effect in the middle to deeper levels of the biofilm.

\section{DISCUSSION}

The mean thickness of the biofilms formed on enamel discs mounted on healing abutments in vivo over seven days in the three subjects was found to vary from 28-96 $\mu \mathrm{m}$. These values are within a similar range to those found in two studies using enamel surfaces placed in splints, where 


\section{Subject 1}

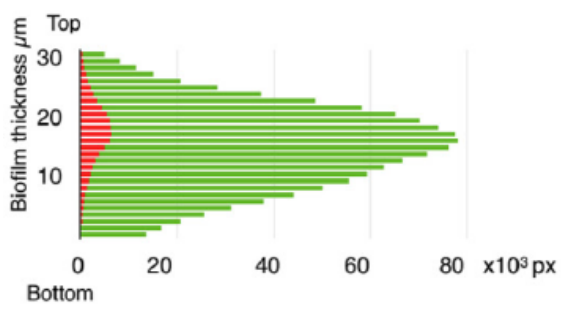

Subject 2

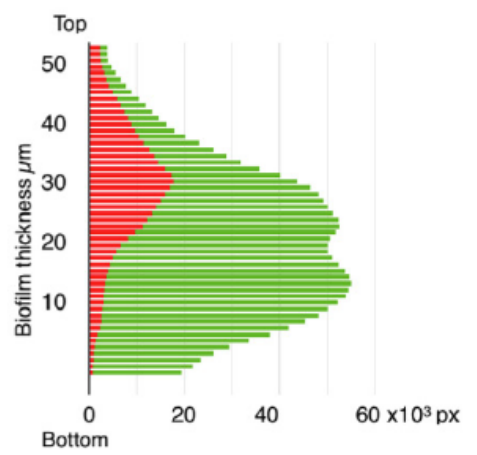

Subject 3

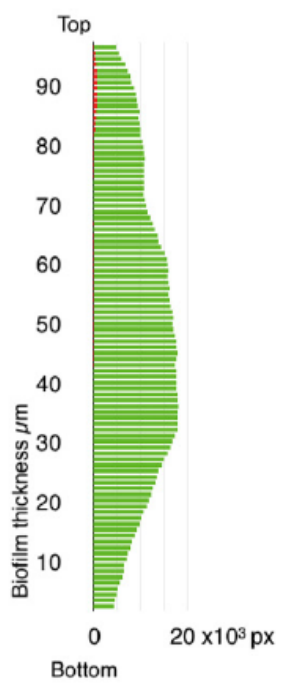

Fig. (4). Bacterial vitality as a function of biofilm depth. To allow assessment of the vitality of bacterial at different levels, z-plane sections were made through the biofilms at $2 \mu \mathrm{m}$ intervals and the number of green and red pixels in each section plotted against biofilm depth.

$\mathrm{CHX}$
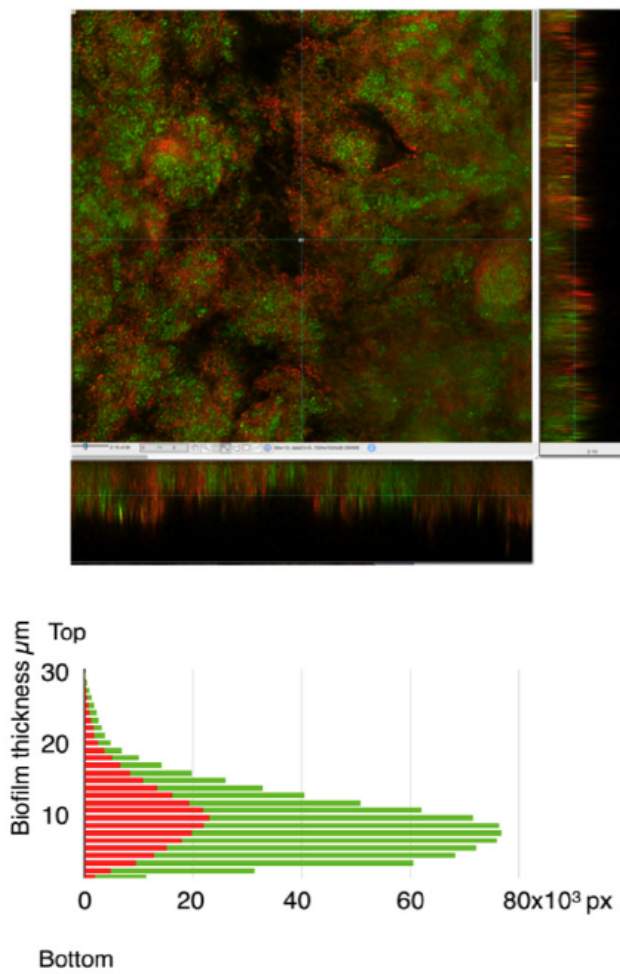

$\mathrm{NaF}$
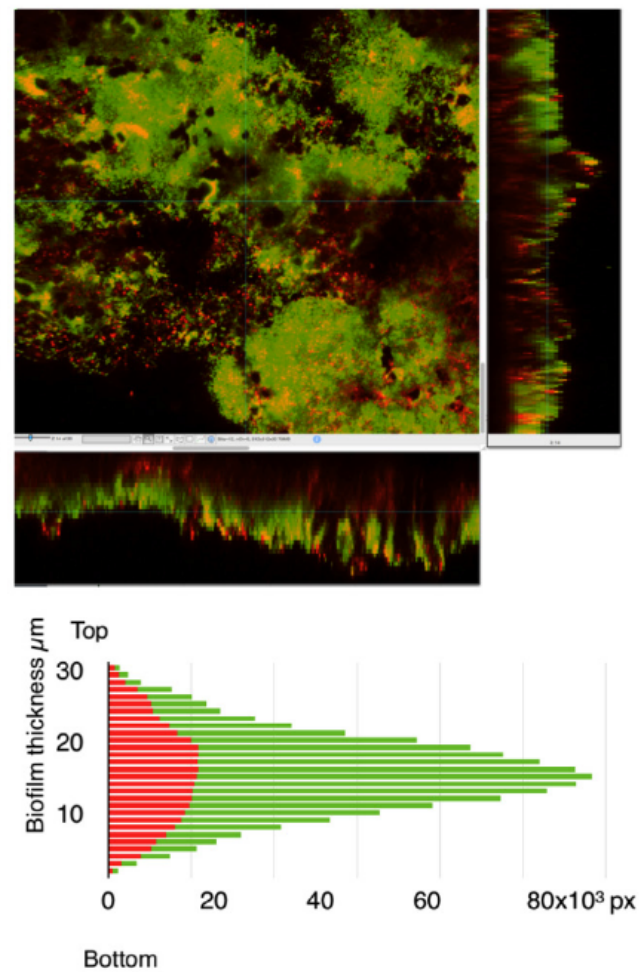

Fig. (5). Effects of CHX and NaF on biofilm bacterial vitality. Biofilms were treated with (a) $0.1 \%$ CHX or (b) $0.2 \% \mathrm{NaF}$ in situ for 1 minute prior to staining with BacLight Live/Dead. Z-plane sections were made and used to generate sagital sections through the biofilms. To allow assessment of the vitality of bacterial at different levels, the number of green and red pixels in each section was plotted against biofilm depth.

biofilm thickness in six individuals after 3 days was $6-45 \mu \mathrm{m}$ [9] or $15-31 \mu \mathrm{m}$ for three participants after 5 days [10]. A recent study using CLSM to observe oral biofilm formation in vivo, showed that plaque deposits formed at the gingival margins after 7 days also had a thickness ranging from a few to $50 \mu \mathrm{m}$ [11]. Taken together, these studies confirm that the biofilms found on enamel surfaces over seven days are mul- tilayered structures, the thickness of which differs between individuals. Apart from the individual variety in thickness, the architecture and distribution of cells within the biofilms differed between subjects. In subjects 1 and 2, the bacterial biomass increased from the enamel surface to the central part of the biofilm and decreased again towards the salivary interface. This is in agreement with the results of a study by Wat- 
son et al. [12] using the Leeds in situ device where the volume of sections occupied by biomass, as shown by staining with toluidine blue, gave a similar picture. Such a profile would suggest a more compact structure in the middle of the biofilm, compatible with, for instance, the presence of mushroom-like structures. In subject 3 however no major variation in biomass relative to depth was seen.

The vitality of the total biomass in the different subjects ranged from $70-98 \%$ suggesting that the handling of the biofilm samples ex vivo did not adversely affect cell survival and that dead and damaged cells make up a small proportion of the biofilms in vivo. In a previous investigation of ex vivo dental plaque samples, mean vitality was shown to range from $80-85 \%$ [13]. In this study, the vitality was always high (96-99\%) in the deepest part of the biofilm closest to the enamel interface. This is in contrast to the results obtained by Auschill et al. [10] where the highest vitality was seen in the middle layers of the biofilm and the study of Netuschil et al. [9] where vital bacteria were found on top of a layer of dead/damaged cells. These differences are difficult to explain but could be attributable to the different models used, where the open surface used here may allow greater diffusion into the biofilms than that which occurs in the splint model used in the previous studies.

Currently, there is a lack of knowledge concerning individual differences in the human oral microbiota. In this study, the composition of the microbiota appeared to vary considerably between individuals with one having mainly spherical cells, probably streptococci, one having mainly filamentous bacteria, most likely Actinomyces, and the third having a mixture of both cell types. These results are in accordance with observations of biofilms from patients using atomic force microscopy where the predominant bacterial forms were also identified as cocci and rods [14]. Both streptococci and actinomycetes are known to be early colonizing species on human enamel surfaces in vivo [1]. Biofilms formed over 48 hours in situ on glass chips placed in splints, examined using 16S $r$ RNA FISH and CLSM, have been found to be dominated by streptococci mixed with filamentous bacteria [15], later identified as for example, Actinomyces naeslundii [16].

When the effect of a one-minute mouth-rinse with $0.2 \%$ $\mathrm{NaF}$ or $0.1 \%$ CHX on biofilms was investigated, the results showed that neither substance removed the biofilm significantly. To our knowledge, no studies on the effects of fluoride rinsing on disruption of established biofilms exist but our data are in agreement with a previous study where no effect of $0.1 \%$ CHX on biofilm biomass was seen $[17,18]$. It is well known that biofilm cells are less susceptible to antimicrobial agents than their planktonic counterparts [3]. In this study, a one-minute rinse with $0.1 \%$ CHX caused a decrease in overall vitality of the biomass from 95 to $63 \%$. The survival rate was thus much greater than for planktonic cells, where no viable counts remained after 5 minutes of CHX exposure $[19,20]$. Dead/damaged cells were seen throughout the biofilm, primarily at the periphery of cell clusters which is in line with a previous study where exposure of $S$. oralis, $S$. gordonii and A. naeslundii biofilms to $0.1 \%$ CHX also affected cells at the edge of clusters [18]. In compact multilayer biofilms, CHX exerted the most prominent effect in the superficial layers [17] suggesting that CHX does not pene- trate well into dense biofilms. The more extensive effect seen here may thus be due to the more open architecture seen in our biofilms. It cannot however, be entirely ruled out that, besides penetration, the effect may be caused by the fact that cells in the centre of clusters have a different phenotype than those at the edges, making them less susceptible to antimicrobial agents [21].

After rinsing with $\mathrm{NaF}$, our results show that the relative distribution of dead/damaged cells was greater in the middle to lower levels of the biofilm, indicating good levels of penetration. Penetration of $\mathrm{NaF}$ has been shown to be comparatively slow but in the presence of saliva, as is the case in our model, the concentration of fluoride in deeper biofilm layers is increased [12]. Bacterial cells are known to take up fluoride, which inhibits a variety of enzymes including enolase as well as dissipating the proton gradient over the cell membrane [22] leading to a reduction in acid production and acid tolerence. Since these metabolic effects may not lead directly to cell damage/death, the positive anti-caries effect of $\mathrm{NaF}$ may be underestimated in our study.

In conclusion, we describe a model that allows the formation of mature, undisturbed oral biofilms on human enamel surfaces in vivo to be combined with qualitative and quantitative ex vivo analyses using techniques such as fluorescent staining and CLSM. Variations in the thickness, architecture, composition and distribution of vital cells between subjects were found. Consistent for all subjects however was the high degree of vitality of cells, particularly in the area closest to the enamel interface. Both $\mathrm{CHX}$ and $\mathrm{NaF}$ caused similar reductions in overall vitality but while the effect of CHX was seen throughout the biofilm, NaF mainly affected cells in the middle to lower layers.

\section{CONFLICT OF INTEREST}

The authors confirm that this article content has no conflict of interest.

\section{ACKNOWLEDGEMENTS}

The study was supported by a grant from the County of Halland, Sweden.

\section{REFERENCES}

[1] Kolenbrander PE, Andersen RN, Blehert DS, Egland PG, Foster JS, Palmer RJ. Communication among oral bacteria. Microbiol Mol Biol Rev 2002; 66: 486-505.

[2] Nyvad B, Killian M. Microbiology of the early colonization of human enamel and root surfaces in vivo. Scand J Dent Res 1987; 95: 369-80.

[3] Costerton JW, Stewart PS, Greenberg EP. Bacterial biofilms: a common cause of persistent infections. Science 1999; 284: 131822.

[4] Marinho VC. Cochrane reviews of randomized trials of fluoride therapies for preventing dental caries. Eur Arch Pediatra Dent 2009; 10: 183-91.

[5] Bradshaw DJ, Marsh PD, Hodgson RJ, Visser JM. Effects of glucose and fluoride on competition and metabolism within in vitro dental bacterial communities and biofims. Caries Res 2002; 36: 816.

[6] Grossmann E, Meckel AH, Isaacs RL, et al. A clinical comparison of antibacterial mothrinses: effects of chlorhexidine, phenolics and sanguinrine on dental plaque and gingivitis. J Periodontol 1989; 60: 435-40 
[7] Petti S, Hausen H. Caries-preventive effect of chlorhexidine gel applications among high-risk children. Caries Res 2006; 40: 51421.

[8] Marsh PD, Keevil CW, McDermid AS, Williamson MI, Ellwood DC. Inhibition by the antimicrobial agent chlorhexidine of acid production and sugar transport in oral streptococcal bacteria. Arch Oral Biol 1983; 28: 233-40.

[9] Netuschil L, Reich E, Unteregger G, Sculean A, Brecx M. A pilot study of confocal laser scanning microscopy for the assessment of undisturbed dental plaque vitality and topography. Arch Oral Biol 1998; 43: 277-85.

[10] Auschill TM, Arweiler NB, Netuschil L, Brecx M, Reich E, Sculean A. Spatial distribution of vital and dead microorganisms in dental biofilms. Arch Oral Biol 2001; 46: 471-6.

[11] Eberhard J, Loewen H, Kruger A, et al. Non-invasive in vivo imaging by confocal laser scanning microscopy of gingival tissues following natural plaque deposition. J Clin Periodontol 2014; 41: 3216.

[12] Watson PS, Pontefract HA, Devine DA, et al. Penetration of fluoride into natural plaque biofilms. J Dent Res 2005; 84: 451-5.

[13] Netuschil L, Reich E, Brecx M. Direct measurement of the bactericidal effect of chlorhexidine on human dental plaque. J Clin Periodontol 1989; 16: 484-8.

[14] Germano F, Bramanti E, Arcuri C, Cecchetti F, Cicciu M. Atomic force microscopy of bacteria from periodontal subgingival biofilm: Preliminary study results. Eur J Dent 2013; 7:152-8.
[15] Dige I, Nilsson H, Kilian M, Nyvad B. In situ identification of streptococci and other bacteria in initial dental biofilm by confocal laser scanning microscopy and fluorescence in situ hybridization. Eur J Oral Sci 2007; 115: 459-67.

[16] Dige I, Raarup MK, Nyengaard JR, Kilian M, Nyvad B. Actinomyces naeslundii in initial biofilm formation. Microbiology 2009; 155: $2116-26$

[17] Takenaka S, Trivedi HM, Corbin A, Pitts B, Stewart PS. Direct visualization of spetial and temporal patterns of antimicrobial action within model oral biofilms. Appl Environ Microbiol 2008; 74: 1869-75.

[18] Corbin A, Pitts B, Parker A, Stewart PS. Antimicrobial penetration and efficacy in an in vitro oral biofilm model. Antimicrob Agents Chemother 2011; 55: 3338-44.

[19] Wilson M, Patel H, Fletcher J. Susceptibility of biofilms of Streptococcus sanguis to chlorhexidine gluconate and cetylpyridinium chloride. Oral Microbiol Immunol 1996; 11: 188-92.

[20] Zaura-Arite E, van Marle, ten Cate JM. Confocal microscopy study of undisturbed and chlorhexidine-treated dental biofilm. J Dent Res 2001; 80: 1436-40.

[21] Fux CA, Costerton JW, Stewrt PS, Stoodley P. Survical strategies of infectious biofilms. Trends Microbiol 2005; 13: 34-40.

[22] Koo H. Strategies to enhance the biological effects of fluoride on dental biofilms. Adv Dent Res 2008; 20: 17-21.

Received: January 26, 2015

Revised: February 21, 2015

Accepted: February 23, 2015

(C) Rabe et al.; Licensee Bentham Open.

This is an open access article licensed under the terms of the Creative Commons Attribution Non-Commercial License (http://creativecommons.org/licenses/by-nc/3.0/) which permits unrestricted, non-commercial use, distribution and reproduction in any medium, provided the work is properly cited. 